\title{
Gamma-irradiated influenza A virus provides adjuvant activity to a co-administered poorly immunogenic SFV vaccine in mice
}

\author{
Rachelle Babb ${ }^{1}$, Jennifer Chan ${ }^{1}$, Jasmine E. Khairat ${ }^{1}$, Yoichi Furuya ${ }^{2 \dagger}$ and Mohammed Alsharifi ${ }^{1}$ * \\ ${ }^{1}$ Vaccine Research Laboratory, School of Molecular and Biomedical Science, Centre for Molecular Pathology, The University of Adelaide, Adelaide, SA, Australia \\ 2 Department of Immunology, The John Curtin School of Medical Research, Australian National University, Canberra, ACT, Australia
}

Edited by:

Murali Krishna Kaja, University of Washington, USA

\section{Reviewed by:}

Ravi Kulkarni, Emory University, USA

Suryaprakash Sambhara, Centers for

Disease Control, USA

*Correspondence:

Mohammed Alsharifi, Vaccine

Research Laboratory, School of

Molecular and Biomedical Science,

Centre for Molecular Pathology, The

University of Adelaide, Adelaide, SA

5005, Australia

e-mail:mohammed.alsharifi@

adelaide.edu.au

${ }^{\dagger}$ Present address:

Yoichi Furuya, Center for Immunology

and Microbial Disease, Albany

Medical College, Albany, NY, USA
Many currently available inactivated vaccines require "adjuvants" to maximize the protective immune responses generated against the antigens of interest. Recent studies in mice with gamma-irradiated influenza A virus ( $\gamma$-FLU) have shown its superior efficacy compared to other forms of inactivated FLU vaccines and its ability to induce both potent interferon type-I (IFN-I) responses and the IFN-I-associated partial lymphocyte activation. Commonly, IFN-I responses induced by adjuvants, combined in vaccine preparations, have been shown to effectively enhance the immunogenicity of the antigens of interest. Therefore, we investigated the potential adjuvant activity of $\gamma$-FLU and the possible effect on antibody responses against co-administrated antigens, using gamma-irradiated Semliki Forest virus ( $\gamma$-SFV) as the experimental vaccine in mice. Our data show that co-vaccination with $\gamma$-FLU and $\gamma$-SFV resulted in enhanced SFV-specific antibody responses in terms of increased titers by sixfold and greater neutralization efficacy, when compared to vaccination with $\gamma$-SFV alone. This study provides promising evidence related to the possible use of $\gamma$-FLU as an adjuvant to poorly immunogenic vaccines without compromising the vaccine efficacy of $\gamma$-FLU.

Keywords: influenza vaccines, combined vaccine, gamma-irradiation, adjuvants, antibodies

\section{INTRODUCTION}

Vaccines represent a fundamental aspect of current control strategies against infectious diseases. However, despite the remarkable success of many vaccines, there still remains many challenges in the field of vaccinology, such as generating effective vaccination strategies against notoriously difficult pathogens like hepatitis $\mathrm{C}$ virus and human immunodeficiency virus (1). Among the various types of currently used vaccines, live attenuated vaccines can mimic natural infections and consequently have been shown to be very effective in generating long-lived immunity. However, due to the high risk of conversion to their highly pathogenic form and the inability to develop live attenuated vaccines for many pathogens, inactivated vaccines such as inactivated whole viruses and purified antigens have typically been used as the main strategy for vaccine design. However, poor immunogenicity of many inactivated vaccines has severely affected their effectiveness. Therefore, inactivated vaccines often require "adjuvants" to enhance their immunogenicity. Importantly, most immunostimulatory adjuvants have been designed to potently stimulate innate signaling pathways through pattern recognition receptors (PRR) such as toll-like receptors (TLR) and cytosolic receptors (2-5). This ultimately leads to the induction of genes encoding for various immune-modulatory molecules including interferon type-I (IFN-I), which stimulate co-stimulatory molecule expression and antigen presentation (6).

Currently in vaccine design, greater attention is being drawn toward designing adjuvants to effectively boost the immune response toward existing vaccine preparations, which fail to induce sufficient immunity. The influence of IFN-I exogenously or through PRR stimulation has been shown to be very effective (7-9). We have previously reported the superiority of gammairradiated influenza virus ( $\gamma$-FLU), compared to other flu vaccine formulations, in terms of inducing cross-protective immunity $(10-12)$. We have also shown that $\gamma$-FLU similarly to its live form is capable of inducing potent IFN-I responses and the associated partial lymphocyte activation $24 \mathrm{~h}$ postchallenge $(13,14)$. Importantly, in contrast to gamma-irradiation of influenza, we have shown that gamma-irradiation of Semliki Forest virus (SFV) abrogates its ability to induce IFN-I responses (15). Therefore, we investigated the potential adjuvant activity of $\gamma$-FLU on co-administered $\gamma$-SFV as an experimental model for poor immunogenic vaccines. Here, we report that co-vaccination with $\gamma$-FLU and $\gamma$-SFV resulted in enhanced SFV-specific antibody titers "by six-folds" when compared to vaccination with $\gamma$-SFV alone. This enhancement in antibody titer was also associated with greater SFV neutralization efficacy and importantly; the vaccine efficacy of $\gamma$-FLU was not affected.

\section{MATERIALS AND METHODS ETHICS STATEMENT}

This study was carried out in strict accordance with the recommendations in the Guide for the Care and Use of Laboratory Animals of The University of Adelaide. The protocol was approved by the 
Animal Ethics Committee at The University of Adelaide (Permit Number: S-2011/119).

\section{VIRUSES AND CELLS}

Avirulent SFV (A7 strain) was grown in vitro by infecting Vero cells using multiplicity of infection (MOI) of 0.1 , and infected flasks were incubated for $24 \mathrm{~h}$ at $37^{\circ} \mathrm{C}$ in a humidified atmosphere with $5 \% \mathrm{CO}_{2}$. Culture supernatants were then collected and clarified to remove cellular debris by centrifugation at $1500 \mathrm{rpm}$ for $5 \mathrm{~min}$. Virus titer was determined by plaque assay on Vero cells to be $3 \times 10^{8} \mathrm{PFU} / \mathrm{ml}$. For the vaccine preparation, SFV stock was concentrated using Millipore filtering devices with $100 \mathrm{kDa}$ cutoff (Millipore) and centrifugation at $2000 \mathrm{rpm}$ for $1 \mathrm{~h}$ at $4^{\circ} \mathrm{C}$ using Eppendorf bench top centrifuge. Virus titer of the concentrated SFV was determined by plaque assay on Vero cells to be $5 \times 10^{8}$ $\mathrm{PFU} / \mathrm{ml}$.

The influenza type A virus, A/PR/8 [(A/Puerto Rico/8/34 (H1N1)], was grown in 10-day-old embryonated chicken eggs (HiChick, SA, Australia). Each egg was injected with $0.1 \mathrm{ml}$ normal saline containing 1 hemagglutination unit (HAU) of virus, incubated for $48 \mathrm{~h}$ at $37^{\circ} \mathrm{C}$, and then held at $4^{\circ} \mathrm{C}$ overnight. The amniotic/allantoic fluids were then harvested, pooled, clarified, and stored at $-80^{\circ} \mathrm{C}$. Gamma-irradiated A/PR8 vaccine preparations were previously prepared by Dr. Furuya at ANU. Briefly, concentrated virus stocks were prepared using chick erythrocytes as previously described (16). Infectious allantoic fluid was incubated with chicken red blood cells (cRBCs) for $45 \mathrm{~min}$ at $4^{\circ} \mathrm{C}$ allowing the hemagglutinin to bind to erythrocytes, and then centrifuged $\left(4^{\circ} \mathrm{C}, 1500 \mathrm{rpm}, 10 \mathrm{~min}\right)$ to remove the allantoic fluid supernatant. The pellets were resuspended in normal saline, incubated for $1 \mathrm{~h}$ at $37^{\circ} \mathrm{C}$ to release the RBCs from the virus, and then centrifuged to remove the erythrocytes and the supernatant containing the virus collected. The titer of the concentrated A/PR8 virus stock $\left(9 \times 10^{8} \mathrm{TCID}_{50} / \mathrm{ml}\right)$ was determined by TCID50 assay (17).

\section{VIRUS INACTIVATION}

Concentrated virus stocks were inactivated by exposure to gammairradiation from a ${ }^{60} \mathrm{Co}$ source [Australian Nuclear Science and Technology Organization (ANSTO) at Lucas Heights/NSW]. A/PR8 and SFV received a dose of 10 and $50 \mathrm{kGy}$, respectively, and they were kept frozen on dry ice during gamma-irradiation. Sterility was tested by two independent methods: plaque assay using MDCK (for A/PR8) or Vero cells (for SFV); and by inoculating embryonated eggs (for A/PR8). The detection limit of our plaque assay is $10 \mathrm{PFU} / \mathrm{ml}$ and no plaque forming unit was detected for the irradiated samples. These tests confirmed sterility of inactivated stocks. In addition, we have estimated the minimum inoculum required to cause a positive infection in embryonated eggs and found that the minimum egg infectious dose that causes detectable HA titers in the allantoic fluid after 2 days of incubation is 0.1 TCID50/egg. Embryonated eggs were inoculated with $100 \mathrm{ml}$ of inactivated preparations per egg and incubated for 2 days at $37^{\circ} \mathrm{C}$ and the allantoic fluid of individual eggs was harvested and tested for virus replication using HA assays. HA titers were negative in the allantoic fluid of these eggs, which illustrates a complete loss of virus infectivity in our inactivated preparations.

\section{MICE AND TREATMENTS}

Wild-type C57B/6 mice (9-10-week-old) were bred under specific pathogen-free conditions and supplied by the Animal Laboratory Services at the University of Adelaide, SA, Australia.

In general, vaccine preparations were diluted using 10-fold serial dilutions and each mouse was injected in the tail vein with $200 \mu \mathrm{l}$ of the relevant virus or vaccine preparation. The following doses were used: live SFV $\left(10^{7} \mathrm{PFU} /\right.$ mouse), $\gamma$-SFV (either $10^{6}, 10^{7}$, or $10^{8} \mathrm{PFU}$ equivalent/mouse), and $\gamma$-FLU $\left(10^{4}, 10^{5}\right.$ TCID50 equivalent/mouse). Refer to text for specific doses used in each experiment. For co-vaccination, the two vaccine preparations were mixed thoroughly in the same tube and administered as a single injection into experimental animals. Vaccination doses are expressed PFU or TCID50 equivalent. In addition, in some experiments Poly(I:C) was injected intravenously at a dose of $150 \mu \mathrm{g}$ in $200 \mu \mathrm{l}$ of PBS per animal as previously reported (18).

\section{ANTIBODY ANALYSIS}

Semliki Forest virus-specific and FLU-specific antibody responses in serum samples were determined by enzyme-linked immunosorbent assay (ELISA). In brief, Maxisorp plates were coated with concentrated SFV or FLU viral antigen diluted in bicarbonate coating buffer $\left(\mathrm{Na}_{2} \mathrm{CO}_{3}, \mathrm{NaHCO}_{3}\right.$, water at $\mathrm{pH}$ 9.6) and incubated overnight at room temperature. Non-specific protein binding sites were then blocked with PBS containing $2 \%$ skim milk powder for $2 \mathrm{~h}$ at room temperature. Fifty microliter volumes of serially diluted serum samples were added to the appropriate wells for $2 \mathrm{~h}$ at room temperature followed by the addition of horse radish peroxidase conjugated goat anti-mouse IgG (Thermo Scientific) at room temperature for $2 \mathrm{~h}$. Plates were developed using TMB peroxidase substrate in the dark for $30 \mathrm{~min}$ and the reaction was stopped with $2 \mathrm{~mol} \mathrm{H}_{2} \mathrm{SO}_{4}$. Absorbance was measured at $450 \mathrm{~nm}$ using a Microplate ELISA reader (Bio-Tek Instruments).

\section{NEUTRALIZATION ASSAYS}

Plaque reduction assay modified from (19) was used to analyze SFV neutralization. Twenty-four well tissue culture plates were seeded with $1.5 \times 10^{5}$ Vero cells/well and incubated overnight at $37^{\circ} \mathrm{C}$ in a humidified atmosphere with $5 \% \mathrm{CO}_{2}$. Aliquots of serum samples from control and vaccinated animals were incubated at $56^{\circ} \mathrm{C}$ for $30 \mathrm{~min}$ to inactivate complements and serially diluted using EMEM media without FCS. Diluted samples were mixed with equivalent amount of DMEM media containing 100 PFU of SFV. Mixtures (sera and virus) were incubated for $1.5 \mathrm{~h}$ at $37^{\circ} \mathrm{C}$ and then used to infect confluent Vero cell monolayer's (in triplicate) prepared earlier. Initially, culturing media was removed from each well prior to addition of virus/serum mixture. Plates were then incubated for $2 \mathrm{~h}$ at $37^{\circ} \mathrm{C}$ to allow infection of monolayers. Following incubation, the infecting mixture was removed and an agar overlay containing $50 \%$ of $1.8 \%$ Bacto-Agar, $40 \%$ DMEM media, $10 \%$ FCS, and $0.002 \%$ Fungizone was added to each well and plates were incubated for 3 days at $37^{\circ} \mathrm{C}, 5 \% \mathrm{CO}_{2}$. Following incubation, cells were fixed with $5 \%$ formalin for $1 \mathrm{~h}$ at room temperature. The overlay was then carefully removed and cell monolayers were stained with $0.2 \%$ crystal violet. Plaques were enumerated to determine the effect of the serum on virus infectivity. 

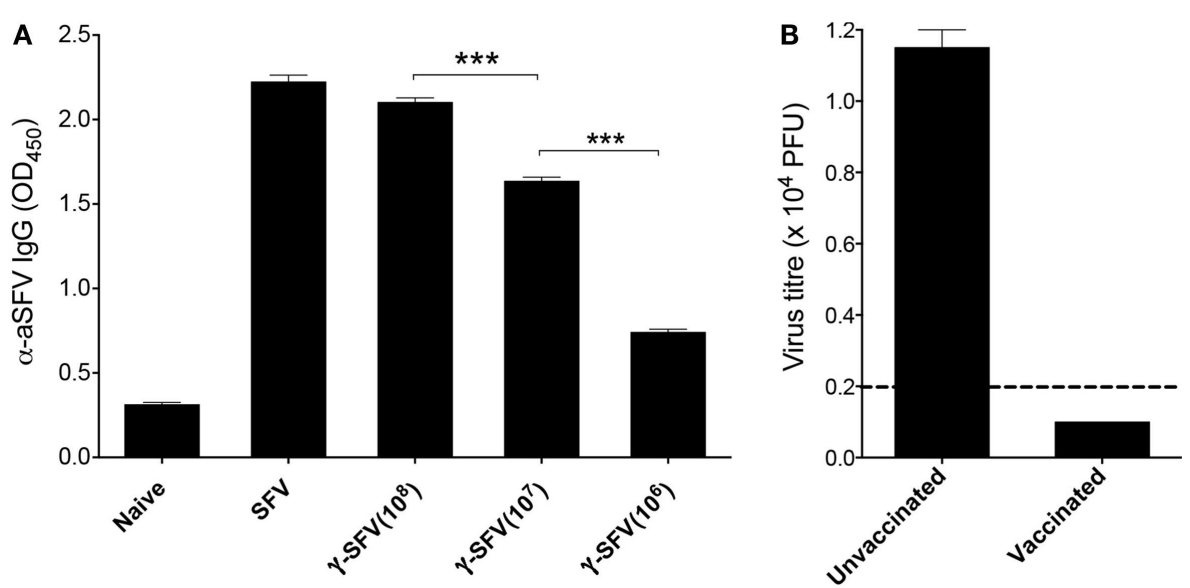

FIGURE $1 \mid \gamma$-SFV vaccine induces protective antibody responses. (A) Mice were injected i.v. with SFV $\left(10^{7}\right.$ PFU) or variable doses of $\gamma$-SFV $\left(10^{6}, 10^{7}\right.$, or $10^{8} \mathrm{PFU}$ equivalent/mouse). Twenty days post-injection, serum SFV-specific IgG levels were measured by direct ELISA using a serum dilution of $1 / 200$. Serum from naive mice served as the negative control. (B) Mice were vaccinated i.v. with $\gamma$-SFV $\left(10^{7}\right.$ PFU equivalent/mouse) and challenged 14 days post-vaccination with SFV (10 $10^{7}$ PFU). Twenty four hours post-challenge, serum SFV titers were determined by plaque assay. Un-vaccinated naive mice infected with SFV served as control. Results are presented as mean $\pm \operatorname{SEM}(n=3)$ and dashed line represents our assay detection limit of 100 PFU, ${ }^{* * *} p<0.001$.
In addition, a hemagglutination inhibition assay (HAI) was used to test the influenza-specific antibody responses, as previously described (17). Aliquots of sera were incubated at $56^{\circ} \mathrm{C}$ to inactivate complements for $30 \mathrm{~min}$, then diluted in PBS containing $1 \%$ RBCs, and left for another $30 \mathrm{~min}$ incubation at room temperature. RBCs within samples were then pelleted by centrifugation at $1400 \mathrm{rpm}$ using a microcentrifuge and supernatants were collected. Twofold serial dilutions were performed using a 96-round well-bottom plate. Fifty microliter of diluted virus (FLU) at a concentration of $80 \mathrm{HAU} / \mathrm{ml}$ was then added to each dilution of sera and incubated for $30 \mathrm{~min}$ at room temperature. PBS containing $1 \%$ RBCs was then added to each well and incubated at $4^{\circ} \mathrm{C}$. Results were analyzed $24 \mathrm{~h}$ later and neutralizing antibodies in each dilution was determined by the presence of a pellet of RBCs at the bottom of the wells.

\section{STATISTICAL ANALYSIS}

Results were expressed as mean \pm SEM. Statistical significance among samples was calculated using an unpaired Student's $t$-test. $P$ values $<0.05$ were considered statistically significant.

\section{RESULTS}

\section{ANTIBODY RESPONSES INDUCED BY $\boldsymbol{\gamma}$-SFV}

Avirulent SFV causes asymptomatic infection characterized by high-titer viremia in adult mice $(20,21)$, and effective viral clearance has been attributed to rapid antibody responses generated by the host (22). Interestingly, primary cytotoxic $\mathrm{T}$ cell responses have been shown to be associated with MHC-I haplotype and restricted to $\mathrm{H}-2^{\mathrm{k}}$ haplotype expressing mice (23). Particularly, C57/B6 mice ( $\mathrm{H}-2^{\mathrm{b}}$ haplotype) have been classified as cytotoxic $\mathrm{T}$ cell non-responders and therefore were used in the study. To confirm that the $\gamma$-SFV vaccination strategies used in this study can generate effective antibody responses, serum SFV-specific IgG levels were measured 20 days post-infection with live SFV $\left(10^{7}\right.$
$\mathrm{PFU} / \mathrm{mouse})$ or vaccination with variable doses of $\gamma$-SFV $\left(10^{6}, 10^{7}\right.$, or $10^{8} \mathrm{PFU}$ equivalent/mouse). Our data illustrate that vaccination with $\gamma$-SFV induces high levels of SFV-specific IgG in the serum of vaccinated mice in a dose-dependent manner (Figure 1A).

To determine if the detected antibody responses are protective, mice were vaccinated with $\gamma$-SFV $\left(10^{7}\right.$ equivalent PFU/mouse $)$ and challenged 21 days later with live SFV $\left(10^{7} \mathrm{PFU} / \mathrm{mouse}\right)$. Twentyfour hours post-challenge, serum samples were tested for virus infectivity by plaque assay. No viral infectivity was detected in all serum samples from previously vaccinated mice in contrast to the high viremia observed in un-vaccinated control mice following a challenge with live SFV (Figure 1B).

It has been previously shown that live SFV infection induces IFN-I and as a consequence promotes Th1 antibody isotype switching (20). Considering the inability of $\gamma$-SFV to induce detectable levels of IFN-I $(13,15)$, the level of IgG isotypes induced by live SFV vs. $\gamma$-SFV was investigated. Mice were infected with SFV $\left(10^{7} \mathrm{PFU} / \mathrm{mouse}\right)$ or vaccinated with $\gamma$-SFV $\left(10^{7}\right.$ equivalent $\mathrm{PFU} / \mathrm{mouse}$ ) and serum SFV-specific IgG1 and IgG2c levels were measured over a time course. Both IgG1 and IgG2c levels appeared to be lower following vaccination with $\gamma$-SFV in comparison to SFV (Figures 2A,B).

\section{THE EFFECT OF CO-ADMINISTRATION OF $\gamma$-FLU AND $\gamma$-SFV ON SFV-SPECIFIC ANTIBODY RESPONSES}

Considering the potent IFN-I responses induced by $\gamma$-FLU $(13,14)$, we investigated the effect of $\gamma$-FLU and $\gamma$-SFV coadministration on SFV-specific antibody responses. Mice were coinjected with $\gamma$-SFV ( $10^{7}$ PFU equivalent/mouse $)$ and $\gamma$-FLU $\left(10^{4}\right.$ or $10^{5}$ equivalent TCID50/mouse) and serum SFV-specific IgG levels were measured at day 20 post-vaccination. Our data illustrate the significant enhancement ( $\sim$ six-folds) of SFV-specific IgG levels in mice co-injected with both $\gamma$-SFV and $\gamma$-FLU compared to mice vaccinated with $\gamma$-SFV alone $(p<0.05)$ (Figures 3A,B). 


\section{A IgG1}

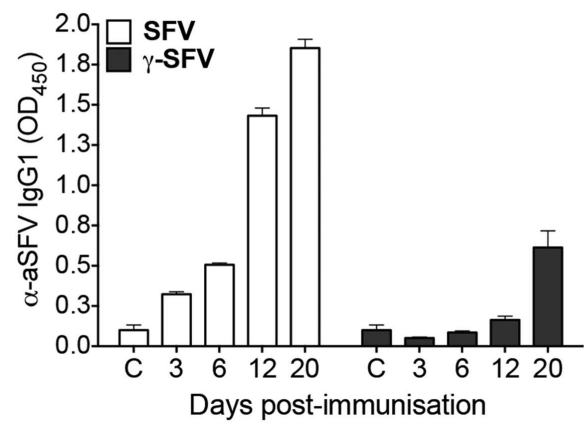

B IgG2C

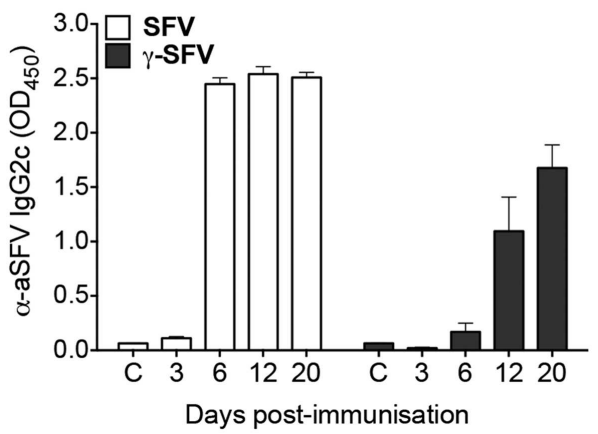

FIGURE 2 | Immunization with $\boldsymbol{\gamma}$-SFV promotes lower antibody levels compared to SFV. Mice were injected i.v. with SFV (10 $\mathrm{PFU})$ or $\gamma$-SFV $\left(10^{7}\right.$ PFU equivalent/mouse). Serum SFV-specific lgG1 (A) and lgG2c (B) levels were measured at $3,6,12$, and 20 days post-immunization by direct ELISA at a serum dilution of $1 / 200$. Sera from naive mice served as negative controls (c). Results are presented as mean $\pm \operatorname{SEM}(n=3),{ }^{* *} p<0.01$.

In addition, we investigated the kinetics of SFV-specific antibody responses post co-administration of $\gamma$-SFV and $\gamma$-FLU. Similarly, our data illustrate the consistent enhancement of SFV-specific antibody responses at all tested time points $(p<0.05)$ (Figure 3C).

To determine whether enhancement of SFV-specific antibody responses mediated by co-administration of $\gamma$-FLU coincides with enhanced SFV neutralization, serial dilutions of the immune sera were tested for their ability to neutralize 100 PFU of live SFV in vitro using a Vero cells based plaque-inhibition assay. Our results clearly illustrate that while immune sera from $\gamma$-SFV alone vaccinated mice have high neutralization activity at $1 / 100$ dilution, this neutralization activity decreased remarkably with every serial dilution to reach $\sim 10 \%$ activity at $1 / 800$ serial dilution. In contrast, immune sera from $\gamma$-SFV and $\gamma$-FLU co-vaccinated mice show significantly higher neutralization values for all tested sera dilutions when compared to sera from $\gamma$-SFV alone vaccinated mice (Figure 3D).

Next, we investigated the effect of $\gamma$-FLU and $\gamma$-SFV coadministration on SFV-specific IgG2c and IgG1 levels and whether co-vaccination may promote a particular IgG isotype. Our data illustrates that co-administration of $\gamma$-FLU and $\gamma$-SFV resulted in significantly enhanced IgG2c levels compared to vaccination with $\gamma$-SFV alone $(p<0.01)$. However, co-vaccination did not lead to significant enhancement in IgG1 responses as the detected SFVspecific IgG1 levels appeared similar in both vaccinated groups (Figures 4A,B).

\section{THE EFFECT OF CO-VACCINATION ON FLU-SPECIFIC ANTIBODY RESPONSES}

Vaccination with $\gamma$-FLU has been shown to elicit homotypic neutralizing antibody responses $(10,11)$. To determine whether the co-administration of $\gamma$-FLU and $\gamma$-SFV affects the host's ability to generate effective FLU-specific humoral responses, we investigated FLU-specific IgG responses at day 20 post-vaccination. Our data indicates that co-vaccination did not suppress the induction of FLU-specific IgG responses induced by $\gamma$-FLU (Figure 5A). We have also tested the neutralizing efficacy of FLU-specific antibodies using HA inhibition assay and our data illustrated that the hemagglutinating activity of $80 \mathrm{HAU}$ of A/PR 8 was inhibited at similar levels by immune sera from mice vaccinated with $\gamma$-FLU alone or mice co-vaccinated with $\gamma$-FLU and $\gamma$-SFV (Figure 5B).

\section{THE EFFECT OF AN IFN-I INDUCING ADJUVANT ON THE IMMUNOGENICITY OF $\boldsymbol{\gamma}$-SFV}

In line with the common approaches used for adjuvant design, the adjuvant activity of $\gamma$-FLU is expected to be related to its ability to induce potent IFN-I responses and the associated IFN-I-mediated partial lymphocyte activation (13). To illustrate the effect of an IFN-I inducing adjuvant on the immunogenicity of $\gamma$-SFV, we evaluated the effect of poly(I:C) and $\gamma$-SFV co-administration on SFV-specific antibody responses. The ability of poly(I:C) to induce IFN-I and its potential use as an adjuvant to enhance humoral responses toward poorly immunogenic proteins has been well-documented $(4,7,24)$. Therefore, mice were vaccinated with $\gamma$-SFV with or without co-injection of poly(I:C) and total SFVspecific IgG levels in the immune sera were analyzed at days 3, 6, 12 , and 20 post-vaccination. Our data illustrate that co-injection of poly(I:C) and $\gamma$-SFV resulted in a significant enhancement in the level of SFV-specific IgG titers in the serum at all time points compared to the injection of $\gamma$-SFV alone (Figure 6).

\section{DISCUSSION}

There has always been an increased demand for safe and effective vaccines to reduce the morbidity and mortality associated with particular viral infections. Non-living antigens are often employed in vaccine strategies and many are poor immunogens (2). We have reported previously the efficacy of $\gamma$-FLU to generate protective immunity upon homotypic and heterosubtypic influenza A virus challenges $(10,25)$. In addition, we have demonstrated the ability of the $\gamma$-FLU vaccine to induce potent IFN-I responses and the associated partial systemic lymphocyte activation $(13,14)$. It has been illustrated previously that IFN-I plays a very influential role in the development of B lymphocytes and consequently antibody production $(26,27)$. In addition, we have reported that $\gamma$-SFV, in contrast to live SFV, does not induce detectable levels of IFN-I (13, 15). Therefore, we used $\gamma$-SFV vaccine as an experimental model to test the adjuvant activity of $\gamma$-FLU.

In general, adjuvants are often used to achieve qualitative/quantitative differences in the immune responses that may include increasing the speed of an immunological response, which 

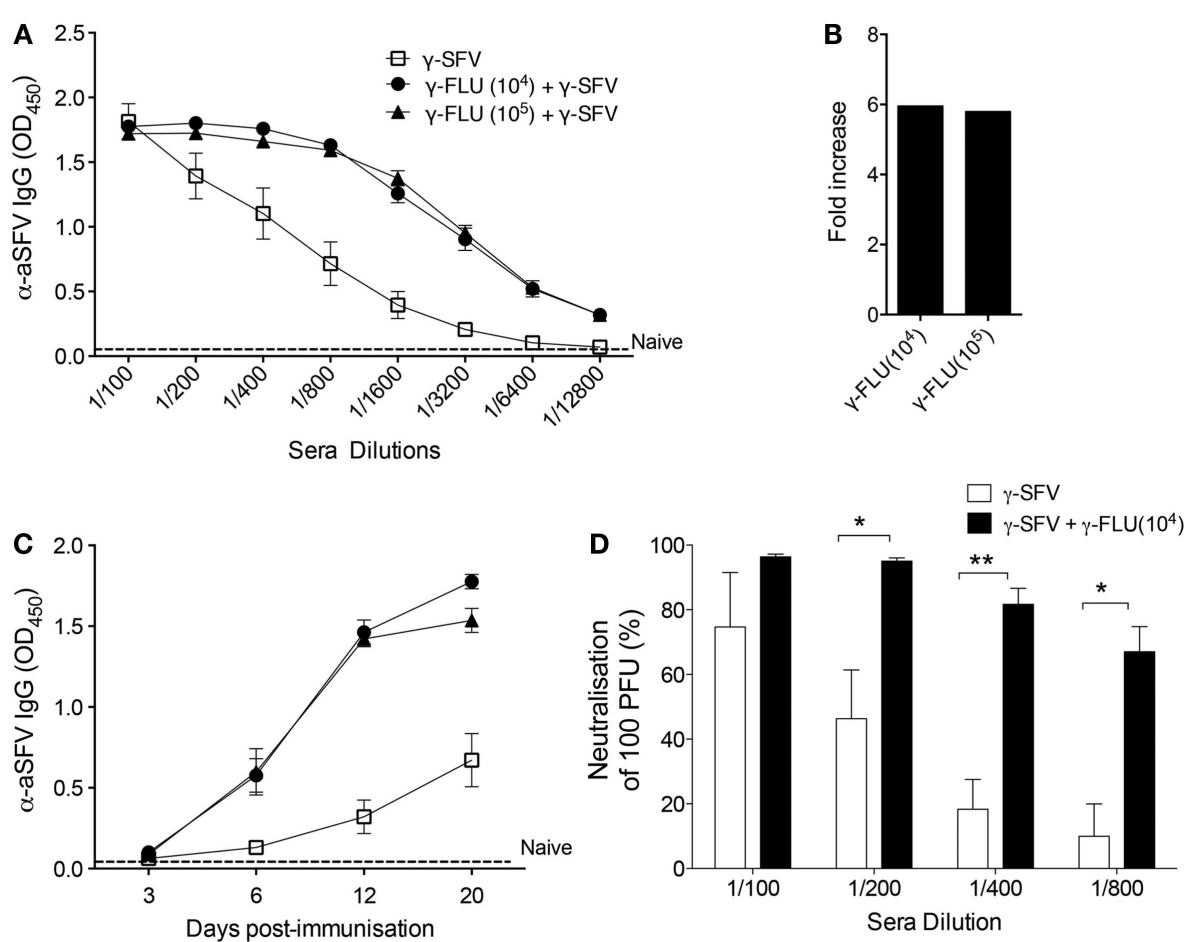

FIGURE 3 | Co-administration of $\boldsymbol{\gamma}$-FLU and $\boldsymbol{\gamma}$-SFV enhances SFV-specific antibody responses. Mice were injected i.v. with a single dose of $\gamma$-SFV $\left(10^{7}\right.$ PFU equivalent/mouse) or co-injected with various doses of $\gamma$-FLU $\left(10^{4}, 10^{5}\right.$ TCID50 equivalent/mouse). (A) Serum SFV-specific IgG concentrations 20 days post-vaccination were analyzed at twofold sera dilutions by direct ELISA and serum from naive mice served as the negative control (naïve).
(B) Folds increase in SFV-specific lgG antibody titers based on the absorbent value of 0.5 for the tested serum dilutions (A). (C) Serum SFV-specific lgG levels were analyzed by direct ELISA using a serum dilution of $1 / 800$ at 3, 6, 12 , and 20 days post-vaccination. (D) Neutralization of SFV by the immune sera collected at day 20 post-vaccination as determined by plaque reduction assay. Data represent mean $\pm \operatorname{SEM}(n=3),{ }^{*} p<0.05,{ }^{*}{ }^{*} p<0.01$.

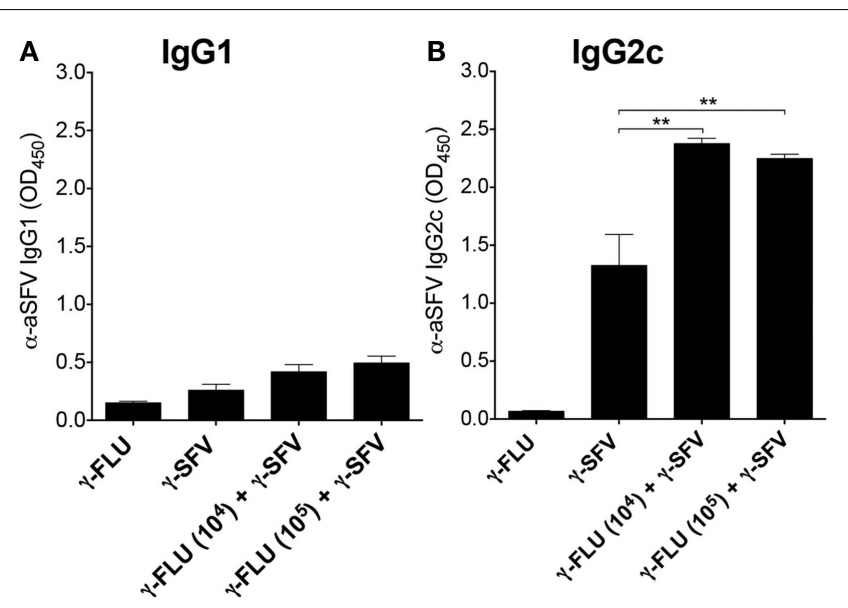

FIGURE 4 | Co-administration of $\gamma$-FLU and $\gamma$-SFV enhances

SFV-specific IgG2c responses. Mice were injected i.v. with a single dose of $\gamma$-SFV (10 7 PFU equivalent/mouse) or co-injected with various doses of $\gamma$-FLU $\left(10^{4}, 10^{5}\right.$ TCID50 equivalent/mouse). SFV-specific lgG1 (A) and IgG2C (B) levels in the serum at day 20 post-vaccination were analyzed by direct ELISA using a serum dilution of $1 / 200$. Sera from $\gamma$-FLU vaccinated mice served as negative controls. Data represent mean $\pm \operatorname{SEM}(n=3){ }^{* *} p<0.01$. is important especially during pandemic outbreaks $(28,29)$. Adjuvants have also been employed to promote specific types of immunity, which may not be efficiently generated by the non-adjuvanted antigens, i.e., Th1 vs. Th 2 cells, $\mathrm{CD}_{8}^{+}$vs. $\mathrm{CD} 4^{+}$cells, and specific types of antibody isotypes (2). Our data clearly show that coadministration of $\gamma$-SFV with $\gamma$-FLU amplified SFV-specific IgG levels with an overall enhancement of $\sim$ six-folds. The enhanced titers observed at day 6 post co-vaccination were equal to the titers detected at day 20 following vaccination with $\gamma$-SFV alone. Thus, confirming an earlier induction and amplification of SFV humoral responses. We have also shown this enhancement to be associated with increased efficiency in virus neutralization. Furthermore, our data illustrate that $\gamma$-FLU promotes enhancement of type- 1 antibody response to co-administered $\gamma$-SFV, as illustrated by the significant increase in IgG2c but not IgG1. This outcome is commonly desired within vaccine development due to the competent functions of IgG2a during antigen clearance relative to IgG1 (30-32).

In line with the many approaches used for adjuvant design, IFN-I responses induced by $\gamma$-FLU may have played an important role in the observed adjuvant activity of $\gamma$-FLU. To evaluate the role of IFN-I, the adjuvant activity of Poly IC on antibody responses to $\gamma$-SFV was investigated. The enhancement of 

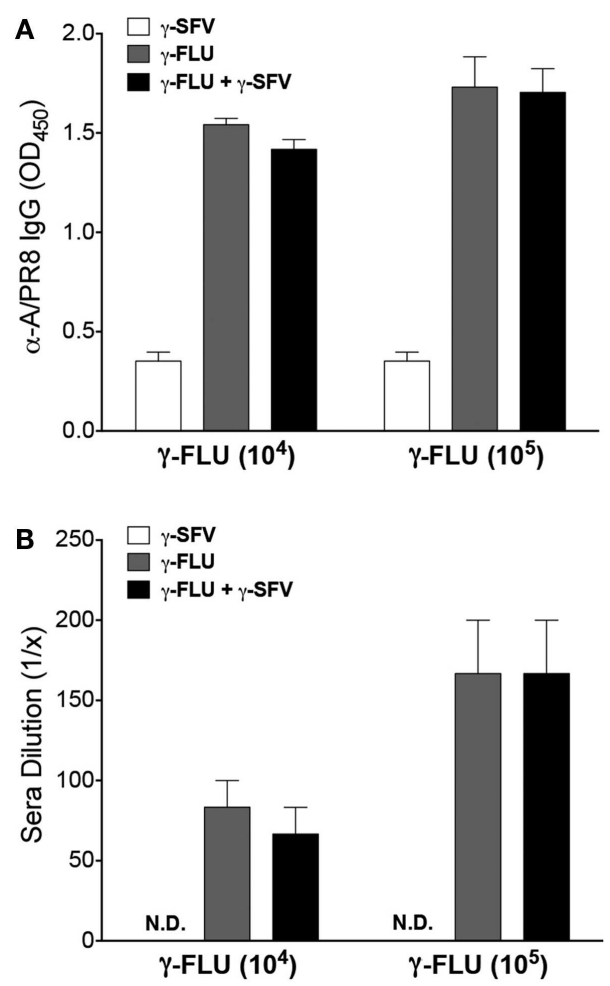

FIGURE 5 |The effect of co-vaccination on FLU-specific antibody responses. (A) Mice were injected i.v. with $\gamma$-FLU (10 ${ }^{4}$ or $10^{5} \mathrm{TCID50}$ equivalent/mouse) or co-injected with $\gamma$-SFV $\left(10^{7} \mathrm{PFU}\right.$ equivalent/mouse) and serum FLU-specific IgG concentrations were analyzed at day 20 post-vaccination by direct ELISA using a serum dilution of $1 / 200$. Sera from $\gamma$-SFV injected mice served as negative controls. (B) HA inhibition assay showing dilution of the immune sera capable of inhibiting the hemagglutinating activity of $80 \mathrm{HAU}$ of A/PR8. Results are presented as mean $\pm \operatorname{SEM}(n=3)$

humoral responses against poorly immunogenic proteins using Poly I:C is well-documented $(4,7,24)$. Poly IC, is a synthetic analog of dsRNA, which is commonly used to stimulate TLR3 and MDA-5 to induce IFN-I and other Th1 priming cytokines such as IL-12 $(8,33,34)$. Consistent with previous reports, our data show that co-immunization with Poly IC and $\gamma$-SFV resulted in significantly enhanced SFV-specific IgG titers relative to titers observed following vaccination with $\gamma$-SFV alone. While this suggest a possible role of IFN-I, more work will be conducted to analyze the underline mechanisms for the adjuvant activity of $\gamma$-FLU.

The ultimate goal of using $\gamma$-FLU as an adjuvant is to exploit the efficacy of the $\gamma$-FLU-mediated immune response toward combined vaccines, in addition to conferring protection against the influenza virus. Our results show that co-administration of $\gamma$-FLU and $\gamma$-SFV did not affect the titers of FLU-specific IgG and did not affect the neutralizing activity of FLU-specific antibodies. Therefore, humoral responses generated against FLU antigens were not hindered when a second vaccine is present in the environment. Overall, this study is a proof-of-concept illustrating that $\gamma$-FLU, a whole virus killed influenza vaccine, can potentially be employed as an adjuvant to increase the quality and magnitude of immune

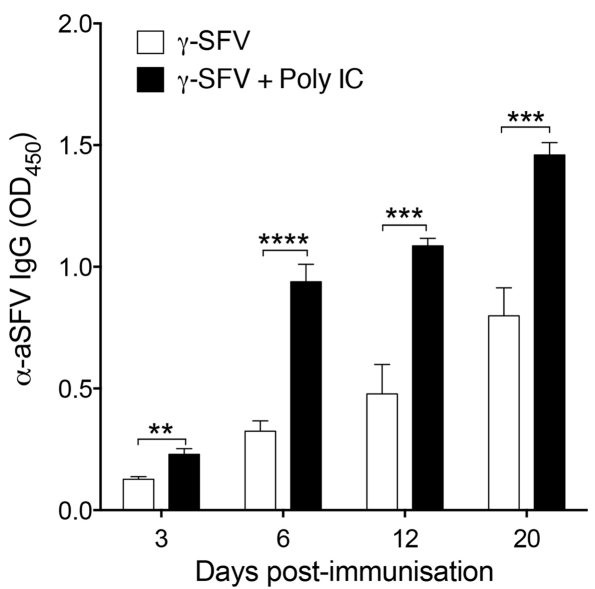

FIGURE 6 | Co-administration of Poly(I:C) and $\boldsymbol{\gamma}$-SFV enhances SFV-specific IgG levels. Mice were vaccinated i.v. with $\gamma$-SFV $\left(10^{7}\right.$ PFU equivalent/mouse) or co-injected with poly (I:C) $(150 \mu \mathrm{g})$. Total SFV-specific $\lg$ levels in the serum were analyzed at day 3, 6, 12, and 20 post-vaccination using direct ELISA using a serum dilution of $1 / 200$. Sera from naive mice served as negative controls. Results are presented as mean $\pm \operatorname{SEM}(n=3)\left({ }^{*}\right)$ denotes statistical significance, ${ }^{*} p<0.01$, ${ }^{* * *} p<0.001$.

responses toward co-administered less immunogenic vaccines. Future studies will investigate the clinical relevance of the $\gamma$-FLUbased combined vaccination strategy, particularly in relation to intranasal and intramuscular routes of administration. Future studies will also examine the effect of co-administered vaccines on the ability of $\gamma$-FLU to induce cross-protective immunity.

\section{REFERENCES}

1. Azad N, Rojanasakul Y. Vaccine delivery - current trends and future. Curr Drug Deliv (2006) 3:137-46. doi:10.2174/156720106776359249

2. Coffman RL, Sher A, Seder RA. Vaccine adjuvants: putting innate immunity to work. Immunity (2010) 33:492-503. doi:10.1016/j.immuni.2010.10.002

3. Wille-Reece U, Flynn BJ, Lore K, Koup RA, Miles AP, Saul A, et al. Toll-like receptor agonists influence the magnitude and quality of memory $\mathrm{T}$ cell responses after prime-boost immunization in nonhuman primates. J Exp Med (2006) 203:1249-58. doi:10.1084/jem.20052433

4. Le Bon A, Schiavoni G, D’Agostino G, Gresser I, Belardelli F, Tough DF. Type I interferons potently enhance humoral immunity and can promote isotype switching by stimulating dendritic cells in vivo. Immunity (2001) 14:461-70. doi:10.1016/S1074-7613(01)00126-1

5. Wu CC, Hayashi T, Takabayashi K, Sabet M, Smee DF, Guiney DD, et al. Immunotherapeutic activity of a conjugate of a Toll-like receptor 7 ligand. Proc Natl Acad Sci U S A (2007) 104:3990-5. doi:10.1073/pnas.0611624104

6. O'Neill LA, Bowie AG. Sensing and signaling in antiviral innate immunity. Curr Biol (2010) 20:R328-33. doi:10.1016/j.cub.2010.01.044

7. Bracci L, Canini I, Puzelli S, Sestili P, Venditti M, Spada M, et al. Type I IFN is a powerful mucosal adjuvant for a selective intranasal vaccination against influenza virus in mice and affects antigen capture at mucosal level. Vaccine (2005) 23:2994-3004. doi:10.1016/j.vaccine.2004.12.006

8. Longhi MP, Trumpfheller C, Idoyaga J, Caskey M, Matos I, Kluger C, et al. Dendritic cells require a systemic type I interferon response to mature and induce CD4+ Th1 immunity with poly IC as adjuvant. J Exp Med (2009) 206:1589-602. doi:10.1084/jem.20090247

9. Haas T, Metzger J, Schmitz F, Heit A, Muller T, Latz E, et al. The DNA sugar backbone $2^{\prime}$ deoxyribose determines toll-like receptor 9 activation. Immunity (2008) 28:315-23. doi:10.1016/j.immuni.2008.01.013 
10. Alsharifi M, Furuya Y, Bowden TR, Lobigs M, Koskinen A, Regner M, et al. Intranasal flu vaccine protective against seasonal and $\mathrm{H} 5 \mathrm{~N} 1$ avian influenza infections. PLoS One (2009) 4:e5336. doi:10.1371/journal.pone.0005336

11. Furuya Y, Chan J, Regner M, Lobigs M, Koskinen A, Kok T, et al. Cytotoxic $\mathrm{T}$ cells are the predominant players providing cross-protective immunity induced by $\{$ gamma $\}$-irradiated influenza A viruses. J Virol (2010) 84:4212-21. doi:10.1128/JVI.02508-09

12. Alsharifi M, Mullbacher A. The gamma-irradiated influenza vaccine and the prospect of producing safe vaccines in general. Immunol Cell Biol (2010) 88:103-4. doi:10.1038/icb.2009.81

13. Furuya Y, Chan J, Wan EC, Koskinen A, Diener KR, Hayball JD, et al. Gammairradiated influenza virus uniquely induces IFN-I mediated lymphocyte activation independent of the TLR7/MyD88 pathway. PLoS One (2011) 6:e25765. doi:10.1371/journal.pone.0025765

14. Alsharifi M, Regner M, Blanden R, Lobigs M, Lee E, Koskinen A, et al. Exhaustion of type I interferon response following an acute viral infection. J Immunol (2006) 177:3235-41. doi:10.4049/jimmunol.177.5.3235

15. Alsharifi M, Lobigs M, Regner M, Lee E, Koskinen A, Mullbacher A. Type I interferons trigger systemic, partial lymphocyte activation in response to viral infection. J Immunol (2005) 175:4635-40. doi:10.4049/jimmunol.175.7.4635

16. Sheffield FW, Smith W, Belyavin G. Purification of influenza virus by red-cell adsorption and elution. Br J Exp Pathol (1954) 35:214-22.

17. Cottey R, Rowe CA, Bender BS. Influenza virus. Curr Protoc Immunol (2001) Chapter 19:Unit1911. doi:10.1002/0471142735.im1911s42

18. Sprent J, Zhang X, Sun S, Tough D. T-cell proliferation in vivo and the role of cytokines. Philos Trans R Soc Lond B Biol Sci (2000) 355:317-22. doi:10.1098/rstb.2000.0568

19. Cohen BJ, Audet S, Andrews N, Beeler J. Plaque reduction neutralization test for measles antibodies: description of a standardised laboratory method for use in immunogenicity studies of aerosol vaccination. Vaccine (2007) 26:59-66. doi:10.1016/j.vaccine.2007.10.046

20. Fazakerley JK, Pathak S, Scallan M, Amor S, Dyson H. Replication of the A7(74) strain of Semliki Forest virus is restricted in neurons. Virology (1993) 195:627-37. doi:10.1006/viro.1993.1414

21. Pusztai R, Gould EA, Smith H. Infection patterns in mice of an avirulent and virulent strain of Semliki Forest virus. Br J Exp Pathol (1971) 52: 669-77.

22. Amor S, Scallan MF, Morris MM, Dyson H, Fazakerley JK. Role of immune responses in protection and pathogenesis during Semliki Forest virus encephalitis. J Gen Virol (1996) 77(Pt 2):281-91. doi:10.1099/0022-1317-77-2-281

23. Mullbacher A, Blanden RV. Murine cytotoxic T-cell response to alphavirus is associated mainly with H-2D (k). Immunogenetics (1978) 7:551-61. doi:10. 1007/BF01844044

24. Arico E, Robertson K, Allen D, Ferrantini M, Belardelli F, Nash AA. Humoral immune response and protection from viral infection in mice vaccinated with inactivated MHV-68: effects of type I interferon. J Interferon Cytokine Res (2002) 22:1081-8. doi:10.1089/10799900260442502

25. Furuya Y, Regner M, Lobigs M, Koskinen A, Mullbacher A, Alsharifi M. Effect of inactivation method on the cross-protective immunity induced by whole 'killed' influenza A viruses and commercial vaccine preparations. J Gen Virol (2010) 91:1450-60. doi:10.1099/vir.0.018168-0
26. Estes DM, Tuo W, Brown WC, Goin J. Effects of type I/type II interferons and transforming growth factor-beta on B-cell differentiation and proliferation. Definition of costimulation and cytokine requirements for immunoglobulin synthesis and expression. Immunology (1998) 95:604-11. doi:10.1046/j.1365-2567. 1998.00645.x

27. Jego G, Palucka AK, Blanck JP, Chalouni C, Pascual V, Banchereau J. Plasmacytoid dendritic cells induce plasma cell differentiation through type I interferon and interleukin 6. Immunity (2003) 19:225-34. doi:10.1016/S1074-7613(03) 00208-5

28. Galli G, Hancock K, Hoschler K, Devos J, Praus M, Bardelli M, et al. Fast rise of broadly cross-reactive antibodies after boosting long-lived human memory B cells primed by an MF59 adjuvanted prepandemic vaccine. Proc Natl Acad Sci US A (2009) 106:7962-7. doi:10.1073/pnas.0903181106

29. Huleatt JW, Jacobs AR, Tang J, Desai P, Kopp EB, Huang Y, et al. Vaccination with recombinant fusion proteins incorporating Toll-like receptor ligands induces rapid cellular and humoral immunity. Vaccine (2007) 25:763-75. doi:10.1016/j.vaccine.2006.08.013

30. Nussenzweig RS, Merryman C, Benacerraf B. Electrophoretic separation and properties of mouse antihapten antibodies involved in passive cutaneous anaphylaxis and passive hemolysis. J Exp Med (1964) 120:315-28. doi:10.1084/jem. 120.2 .315

31. Unkeless JC. The presence of two Fc receptors on mouse macrophages: evidence from a variant cell line and differential trypsin sensitivity. J Exp Med (1977) 145:931-45. doi:10.1084/jem.145.4.931

32. Vogel FR. Improving vaccine performance with adjuvants. Clin Infect Dis (2000) 30(Suppl 3):S266-70. doi:10.1086/313883

33. Alexopoulou L, Holt AC, Medzhitov R, Flavell RA. Recognition of doublestranded RNA and activation of NF-kappaB by Toll-like receptor 3. Nature (2001) 413:732-8. doi:10.1038/35099560

34. Davey GM, Wojtasiak M, Proietto AI, Carbone FR, Heath WR, Bedoui S. Cutting edge: priming of CD8 $\mathrm{T}$ cell immunity to herpes simplex virus type 1 requires cognate TLR3 expression in vivo. J Immunol (2010) 184:2243-6. doi:10.4049/jimmunol.0903013

Conflict of Interest Statement: The authors declare that the research was conducted in the absence of any commercial or financial relationships that could be construed as a potential conflict of interest.

Received: 02 April 2014; accepted: 24 May 2014; published online: 10 June 2014. Citation: Babb R, Chan J, Khairat JE, Furuya $Y$ and Alsharifi $M$ (2014) Gamma-irradiated influenza A virus provides adjuvant activity to a coadministered poorly immunogenic SFV vaccine in mice. Front. Immunol. 5:267. doi: 10.3389/fimmu.2014.00267

This article was submitted to Microbial Immunology, a section of the journal Frontiers in Immunology.

Copyright (c) 2014 Babb, Chan, Khairat, Furuya and Alsharifi. This is an open-access article distributed under the terms of the Creative Commons Attribution License (CC $B Y)$. The use, distribution or reproduction in other forums is permitted, provided the original author(s) or licensor are credited and that the original publication in this journal is cited, in accordance with accepted academic practice. No use, distribution or reproduction is permitted which does not comply with these terms. 